\title{
Importancia de la probabilidad y la estadística en la formación del Ingeniero
}

Importance of the probability and the statistics in the formation of the engineer

\section{MARÍA ALEJANDRA OSORIO-ANGARITA}

Ingeniera de Sistemas

MSc. en Matemáticas

Grupo de Investigación GIPROCAS

Universidad de Boyacá, Colombia aosorio@uniboyaca.edu.co

\section{AUGUSTO BIMBERTO SUÁREZ-PARRA}

Licenciado en Matemáticas y Estadística

Máster en Bioestadística

Grupo de Investigación GIPROCAS

Universidad de Boyacá, Colombia

augustosuarez@uniboyaca.edu.co

Recibido: 25/06/2012

Aceptado: 17/10/2013 


\title{
RESUMEN
}

Los ingenieros se ven enfrentados generalmente a la toma de decisiones en situaciones en donde la incertidumbre, el azar y el riesgo, son relevantes. La formación en probabilidad y estadística es importante para su desempeño laboral. Se presenta una breve descripción de las técnicas estadísticas de mayor uso para generar información útil en la toma de decisiones, junto con algunas áreas y disciplinas que guardan relación con la ingeniería, para las cuales la probabilidad y la estadística tienen aplicación.

Palabras Clave: Estadística y probabilidad, Ingeniería, Método de Ingeniería, Pensamiento aleatorio, Pensamiento estadístico.

\begin{abstract}
Engineering does have to deal with the solution of problems that involve uncertainty and risk. The main tool that they use to solve them is the use of probability and statistics. This paper presents a description of the most common statistical methods that are used by engineers to analyze and solve problems.
\end{abstract}

KeyWords: Statistics and Probability, Engineering, Engineering method, Random Think, Statistical Think. 


\section{INTRODUCCIÓN}

La Educación Superior pretende preparar al estudiante para enfrentar diversos desafíos laborales, los cuales en realidad son de naturaleza indeterminada y cuya manera de afrontarlos puede ser compleja. Ante esta perspectiva, como lo afirma Viles (2007), el profesional de la Ingeniería debe ser consciente que la variabilidad es un fenómeno presente en los procesos y que su control es posible efectuarlo mediante el uso de la Estadística en el diseño y desarrollo de nuevos productos, así como en el control y la mejora de la calidad de los procesos de producción. En este trasegar, el ingeniero no puede tomar decisiones siguiendo solo su intuición, sino que debe basarse en hechos reales, utilizar las herramientas que le ofrecen las ciencias y valerse de estrategias para la toma de decisiones en diferentes escenarios.

En el presente artículo se muestra la importancia que tiene el pensamiento aleatorio y estadístico para el ingeniero, como responsable del planteamiento de soluciones a problemas que se presentan en las organizaciones. Igualmente, se hace una descripción de algunas técnicas estadísticas usadas frecuentemente como el control estadístico de calidad, los estudios de confiabilidad y pruebas de vida, el análisis y diseño de experimentos, el análisis de regresión, las series de tiempo, el análisis exploratorio de datos, la simulación y el muestreo, con las cuales el ingeniero, además de obtener información para la toma de decisiones y la solución de problemas, puede comprender e implementar políticas para el mejoramiento de productos y procesos, adquiriendo una ventaja competitiva en el mundo laboral.

\section{RELEVANCIA DEL APRENDIZAJE DE LA PROBABILIDAD Y DE LA ESTADÍSTICA EN LOS PROGRAMAS DE INGENIERÍA}

Muchas características asociadas a fenómenos naturales, sociales, de la cotidianidad de las personas y al mundo productivo de las empresas, ocurren de manera aleatoria, por lo que su estudio se convierte en una labor compleja. Esta es una razón para que la universidad continúe con la tarea, ya iniciada en la educación básica y media, de preparar a los futuros profesionales en pensamiento aleatorio, estocástico o probabilístico, entendido como "aquel que ayuda a tomar decisiones en situaciones de incertidumbre, de azar, de riesgo o de ambigüedad por falta de información confiable, y en las que no es posible predecir con seguridad lo que va a pasar" (Ministerio de Educación Nacional - MEN, 2006, p.64), de tal manera que sean profesionales más eficientes a la hora de solucionar problemas bajo incertidumbre. 
Para Agnelli (2009), la enseñanza de la Probabilidad queda justificada por su relevancia en la cotidianidad de las personas, por su importancia en el marco de la historia de las ideas científicas, por sus complejas relaciones entre intuiciones y teorías normativas y por su relación entre teorías y aplicaciones. En el campo de la Ingeniería, Behar, et al, (2002) afirman que para este profesional la cuantificación del riesgo implica conocer elementos básicos de probabilidad y a partir de un evento simple, se tenga la capacidad de valorar el riesgo de eventos con mayor complejidad. Por ejemplo, en la industria muchos de los eventos que se presentan son de esta naturaleza y su tratamiento es un reto que se debe enfrentar.

La Estadística por su parte se ha convertido en las últimas décadas, en una ciencia importante, principalmente por tres razones: a) es una herramienta utilizada por muchas disciplinas, ciencias y profesiones para el desarrollo particular de sus investigaciones; b) la competitividad en el sector productivo ha generado una preocupación por darle un tratamiento estadístico a problemas de productividad y calidad en las organizaciones, aspecto que Deming (el padre del desarrollo industrial Japonés) destacó en los siguientes términos: "Ningún recurso es tan escaso como el conocimiento estadístico. No hay conocimiento que pueda contribuir tanto a mejorar la calidad, productividad y competitividad de las empresas como el de los métodos estadísticos" (Romero y Zúnica, 2008, p.19) y c) como lo expone Zapata (2011), la sociedad moderna se encuentra rodeada de datos que proceden de diferentes fuentes y cuyo tratamiento implica que cada persona en su cotidianidad tenga que leer, interpretar, criticar y apropiarse de información, para lo cual se requieren algunas habilidades, las cuales Batanero (2002) enmarca con el término de alfabetización estadística.

Para Navidi (2006), la Estadística se dedica a la recopilación, análisis, e interpretación de datos con incertidumbre y los métodos estadísticos, ayudan a los científicos e ingenieros a diseñar experimentos con cierta validez, para obtener conclusiones confiables a partir de los datos obtenidos; en este punto hay que tener en cuenta lo expuesto por Viles (2007), al considerar que antes de aplicar métodos estadísticos es pertinente comprender la naturaleza y el origen de los datos, los cuales son de poca utilidad para la toma de decisiones, si su validez y confiabilidad está en duda. La aplicación eficaz de la Estadística en la industria redunda en el crecimiento de las organizaciones y no es posible sin el concurso de profesionales idóneos en esta área.

Al relacionar la Probabilidad y la Estadística con los programas de Ingeniería, es pertinente señalar que el profesional en Ingeniería requiere de heurísticas y herramientas que le permiten decidir en ambientes de riesgo e incertidumbre, las cuales según Koen (1985) se pueden encontrar en el contexto laboral de estas disciplinas, además, al caracterizar al ingeniero, expresa cómo éste inicia su trabajo a partir de situaciones inciertas cuyo punto de llegada es aleatorio, lo cual implica que el recorrido que hace para cumplir con su tarea, lo lleva a resolver dificultades y elegir entre varias alternativas aquella que a su juicio tenga el mínimo riesgo. Por su parte para Montgomery y Runge (2004), el ingeniero es un profesional que soluciona problemas de interés para la sociedad con la aplicación de principios 
científicos, tarea cotidiana que realiza bajo el enfoque del método de ingeniería, cuya aplicación contribuye a reducir costos en beneficio de la empresa y los consumidores.

Sin embargo, la aplicación del método de ingeniería no implica certidumbre en los resultados y por el contrario, el ingeniero se encuentra con variabilidad en los datos y una multiplicidad de alternativas de solución; es aquí, donde la Probabilidad y la Estadística dan su aporte para orientar el curso de acción del tomador de decisiones, quien con una sólida formación en teoría de la estimación, en pruebas de hipótesis y en pensamiento estadístico, tiene las herramientas para elegir la mejor alternativa a la luz de los datos.

\section{ALGUNAS TÉCNICAS ESTADÍSTICAS UTILIZADAS EN INGENIERÍA}

El ingeniero, en la tarea de diseñar experimentos y analizar datos de diferentes fuentes, acude a las diversas técnicas estadísticas que dispone con miras a reducir la incertidumbre y generar seguridad para la toma de decisiones. En este propósito su formación en pensamiento estadístico le ayuda a tener mayor efectividad ya que según Wild y Pfannkuch (2007) quienes tengan esta formación son personas que se caracterizan entre otros aspectos porque: a) evitan hacer especulaciones subjetivas y dan solución con base en los datos, b) tienen claridad sobre los procesos de generación de datos, c) involucran la variabilidad y la incertidumbre, d) tienen en cuenta la validez interna y externa en la experimentación, e) conocen e interpretan los procesos de estimación, f) entienden el concepto de significancia estadística, g) valoran la utilidad de la Estadística para estimar, comparar, predecir, construir indicadores y mostrar sus resultados.

Un profesional con estas características es una garantía que tienen las organizaciones para mejorar sus procesos en un mundo competitivo. Si una empresa quiere enriquecer su talento humano en pensamiento estadístico, una de las estrategias, de acuerdo con Carballo (2010), es implementar en las organizaciones proyectos como Seis Sigma, ya que estos van de la mano con la formación de las personas en este tipo de pensamiento.

A continuación se hace una descripción de las técnicas estadísticas que de alguna manera se vale el ingeniero para generar información útil para la toma de decisiones empresariales.

\section{Control estadístico de la calidad}

Una primera aplicación de la Probabilidad y la Estadística al servicio de la Ingeniería surge en el contexto de la productividad y el control de la calidad. Hoy en día las empresas son conscientes que la competitividad guarda relación con la calidad, el servicio y el precio del producto. En este sentido, el control estadístico de calidad para Juran y Gryna (1988), es la aplicación de diferentes técnicas 
estadísticas con el propósito de medir y mejorar la calidad de los procesos. Para dicho control es imprescindible el uso de herramientas estadísticas básicas como diagramas causa-efecto, diagramas de Pareto, diagramas de dispersión, histogramas, hojas de verificación, gráficos y cuadros de control. Hacer estratificación, cuando sea necesario, pretende describir, comprender y controlar la variabilidad, la cual está relacionada con la maquinaria, la mano de obra, las mediciones, el medio ambiente, los métodos y los materiales. Su aplicación implica un compromiso firme por parte del nivel estratégico de la empresa, acompañado de un cambio en la manera de pensar en las personas. Es primordial formar en pensamiento estadístico a funcionarios del nivel táctico de la organización y en el manejo de las herramientas estadísticas a quienes conforman el nivel operativo.

\section{Confiabilidad y pruebas de vida}

Estos estudios se basan en la Probabilidad y la Estadística y son utilizados en la gestión de mantenimiento industrial, la que es considerada una actividad clave para mejorar la calidad y la competitividad de las empresas. Para Gutiérrez y De la Vara (2004), la confiabilidad se relaciona con la probabilidad de que un componente o sistema desempeñe satisfactoriamente la función para la cual fue creado, durante un periodo establecido y en unas condiciones normales de operación especificadas. Los estudios de confiabilidad analizan el comportamiento de vida de un componente o sistema en unidades de tiempo y su utilización en la planeación del mantenimiento permite calcular indicadores de disponibilidad como el tiempo medio entre fallas - MTBF y el tiempo medio de reparación - MTTR, entre otros. Un mantenimiento enfocado en la confiabilidad ayuda a prolongar la vida de los equipos y sistemas en el futuro inmediato, con lo cual se puede garantizar con cierta confianza la funcionalidad de los sistemas y la producción de la empresa.

\section{Diseño de experimentos}

En muchos trabajos de ingeniería el diseño experimental visto como una prueba en la que se inducen cambios deliberados en las variables de un proceso o sistema (Montgomery, 1991), permite con fundamento en la Estadística introducir mejoras en el desempeño de procesos de manufactura y contribuye en la toma de decisiones sobre nuevos productos y procesos. Su aplicación consiste en controlar un conjunto de variables o factores, hacer un análisis de varianza para probar hipótesis y luego determinar el subconjunto de aquellas con mayor influencia en el proceso. Lo anterior lleva al mejoramiento del proceso, la reducción de la variabilidad y la disminución de tiempos y costos.

\section{Técnicas de regresión}

Hay investigaciones en donde es necesario formular un modelo estadístico para establecer una relación funcional entre una variable dependiente o de respuesta y una(s) variable(s) independiente(s), regresora(s) o predictora(s). La técnica consiste en buscar un modelo estadístico de naturaleza lineal o no lineal con el cual se hacen predicciones y se explica la variabilidad que hay en la variable dependiente, 
asociada a la variabilidad de la(s) variable(s) independiente(s). Los parámetros del modelo se pueden estimar a partir de los datos de la muestra, generalmente por el método de mínimos cuadrados, y en algunos casos por el de máxima verosimilitud. Una vez se tiene el modelo de predicción con sus parámetros hay que validarlo y hacer cumplir los supuestos sobre los errores, los cuales en general son los mismos, ya sea en un análisis de regresión simple o múltiple; si es así, sus resultados pueden ser usados para la toma de decisiones (Hair, Anderson, Tatham, Black, 2008).

\section{Series de tiempo}

Al considerar que muchos fenómenos económicos y empresariales presentan variación en el tiempo, puede ser de utilidad para el ingeniero o empresario usar los resultados que proporcionan los modelos de series de tiempo con el fin de hacer predicciones para mejorar la toma de decisiones y contar con una valiosa ayuda en la etapa de planeación empresarial. Para Guerrero (1991), las series de tiempo se relacionan con la medición de características o variables económicas a intervalos de tiempo fijos, para luego estudiar su composición y analizar su evolución en el futuro. La Estadística ayuda a la descripción de las series de tiempo y según el modelo, es de utilidad para probar los supuestos sobre los errores e inferir resultados cuando sea necesario.

\section{Análisis exploratorio de datos - AED}

Es un enfoque situado entre la estadística descriptiva y la inferencial, que privilegia el uso de diferentes representaciones gráficas como diagramas de caja, de dispersión, de puntos, tallos y hojas e histogramas entre otros, lo mismo que cálculos sencillos de Estadística para analizar los datos según su variabilidad y regularidad, buscando la detección de valores atípicos, patrones de comportamiento y posibles hipótesis, con lo cual se preparan los datos para un posterior análisis confirmatorio. Sin desconocer la utilidad que tiene el AED, hay que tener cierta precaución, pues se puede llegar a pensar que la Estadística se limita solo a un análisis de este tipo, desaprovechando los aportes que hace esta ciencia desde otros enfoques (León, 2007).

\section{Muestreo}

En muchos estudios cuando se tiene interés por conocer uno o varios parámetros de una población y no es posible por razones económicas, limitación de tiempo y aspectos prácticos tomar de manera exhaustiva los elementos de la población, se tiene que tomar una muestra que contenga las características de interés para el investigador. Por ejemplo, para conocer algunas características de la calidad del agua o de un producto terminado en una empresa, no se necesita tomar toda el agua de un lago o todos los productos terminados en un día de producción, basta con acudir a las técnicas de muestreo y estimar los parámetros de interés en la población. 
La selección de una muestra se puede hacer por métodos aleatorios o no aleatorios, pero para este artículo se consideran los primeros, ya que satisfacen las condiciones que permiten hacer estimaciones de los parámetros con cierta precisión y control del error de estimación. Para Cochran (2000), un muestreo es aleatorio si satisface las siguientes propiedades: a) se conoce el conjunto de muestras posibles, b) cada muestra tiene una probabilidad de ser seleccionada, c) se selecciona una de las muestras utilizando un método de selección aleatorio y d) se define un método para estimar un único parámetro de la muestra. Los diseños de muestreo básicos son muestreo aleatorio simple, estratificado, sistemático y por conglomerado, los cuales son la base para construir diseños de mayor complejidad según el objeto de estudio.

Es oportuno mencionar que cuando se hace la medición de múltiples características a un objeto, se puede tener como propósito explicar, correlacionar y predecir la asociación, no entre dos variables, sino entre una combinación de múltiples variables o grupos de éstas. Lo anterior implica acudir a los métodos de análisis multivariados de datos, los que se clasifican en métodos factoriales y técnicas de clasificación (Díaz, 2002).

El conocimiento y aplicación de los métodos estadísticos descritos, al igual que las herramientas estadísticas para la calidad ya mencionadas, son características ideales que deben tener los ingenieros para trabajar en función de la calidad y la productividad de las organizaciones.

\section{ALGUNOS CAMPOS DE APLICACIÓN DE LA PROBABILIDAD Y LA ESTADÍSTICA EN PROGRAMAS DE INGENIERÍA}

La Probabilidad y la Estadística tienen aplicación en el contexto de múltiples áreas y disciplinas las cuales son parte integral de diversos programas académicos de formación tecnológica y profesional. Algunas de estas se señalan a continuación teniendo en cuenta su cercanía y utilización en los programas de Ingeniería.

En el campo de la Ingeniería Ambiental y ciencias de la tierra, la Probabilidad y la Estadística tienen una gran aplicación en diferentes situaciones: la caracterización, modelamiento y pronóstico de la calidad del aire y agua; los estudios de caudales y precipitación en hidrología, entre otros, donde se trabaja ya sea con datos que se ajustan a una distribución normal o aquellos que se alejan de esta distribución, y para la estimación de los periodos de retorno de un evento a partir de las probabilidades de ocurrencia de las precipitaciones máximas que se disponen.

Para el Ingeniero Geólogo, según Santillan, Arcos, Vidal y Cruz (2009), la Estadística es útil en su formación por el cotidiano trabajo con variables aleatorias y su aplicación en estudios de suelos por medio de la granulometría, donde se necesita una adecuada interpretación gráfica y estadística para 
comprender científicamente el comportamiento de los procesos actuales y hacer inferencias en los procesos sedimentarios del pasado.

En la salud ocupacional, higiene y seguridad industrial, la Estadística es vital para el registro metódico de los accidentes laborales, el análisis de causalidad, la evaluación de riesgos ocupacionales y para el cálculo de indicadores que soportan la implementación de programas de prevención. En el mismo sentido, la Probabilidad y la Estadística se utiliza para estudiar los fenómenos actuariales, los cuales para López y López (1996), se presentan de manera aleatoria y traen consecuencias económicas, las que si fuesen negativas, hay que evitarlas, o en su defecto tener planes para prevenir las pérdidas que genera el riesgo a la empresa o personas afectadas.

La Probabilidad y la Estadística son base para realizar estudios donde no hay certeza en los datos, como aquellos que se enmarcan en el campo de la Investigación de Operaciones, en los cuales se requiere utilizar técnicas estadísticas o trabajar con modelos probabilísticos, como los relacionados con líneas de espera y pronósticos o en la teoría de la decisión. Igualmente se utiliza en los procesos de planeamiento, control y pronóstico de la producción y en la investigación de mercados, campo que es propicio para la aplicación de los métodos estadísticos multivariados.

Cuando los costos de un experimento sobrepasan los alcances del investigador o hay imposibilidad para trabajar con datos reales, una alternativa es acudir a la simulación, entendida esta como una imitación que se hace de un sistema mediante el uso de un modelo informático, con el propósito de evaluar y mejorar el rendimiento del sistema (Harrell, Ghosh, Bowden, 2003). Para hacer una simulación hay que conocer con la mayor aproximación posible las distribuciones de probabilidad de los datos, por lo tanto, el conocimiento que se tenga en fundamentos matemáticos, Probabilidad y Estadística, es importante para la descripción del fenómeno estudiado. Con la simulación se pueden estimar probabilidades, sesgos, tiempos de vida, promedios y varianzas, lo mismo que probar hipótesis; como base para posteriormente simular procesos.

Además de lo anterior, no se puede dejar sin destacar que la Estadística es una ciencia metodológica básica para el método científico experimental, el cual proporciona información útil en diversos campos como agricultura, diseño de productos y procesos, genética, química farmacéutica, química analítica, procesamiento de imágenes y optimización, entre otros campos, lo mismo que para estudios donde se generan datos por observación como en paleontología y arqueología, demografía, meteorología y estudios de clima, exploración de minerales e hidrocarburos, prevención de desastres naturales, logística, planeamiento y control de inventarios, por citar algunos ejemplos. En general la utilidad de la Estadística llega a cualquier campo donde se tenga que hacer procesamiento de datos.

Estas aplicaciones de la Probabilidad y la Estadística en Ingeniería muestran la necesidad de formar al futuro ingeniero en pensamiento aleatorio y estadístico, puesto que al involucrarlos en su quehacer, 
esta formación le será útil para el éxito en la solución de problemas. Por lo anterior todos los planes de estudio de los programas de Ingeniería contemplan por lo menos dos cursos de Estadística en donde la Probabilidad tiene un espacio reservado. Actualmente se imparten estos cursos a partir de los conceptos elementales de Probabilidad y Estadística que se orientan desde la educación básica y media, como lo establecen los lineamientos y estándares del Ministerio de Educación Nacional (MEN, 1998), (MEN, 2006).

Con todo lo anterior y de acuerdo a lo afirmado por Behar, et al (2002), la formación Estadística no puede ser opcional en el currículo de un ingeniero, sino que debe ser parte esencial de los programas. Trabajar con información incompleta, en ambientes de incertidumbre, es la condición natural del entorno de un ingeniero, quien deberá tener la capacidad de descubrir patrones en medio de la variabilidad y actitud para considerar la incertidumbre como elemento importante en el estudio y solución de problemas ingenieriles.

\section{CONSIDERACIONES FINALES}

Es innegable la importancia que tiene en la industria la aplicación de técnicas estadísticas en función de la productividad, pero este hecho no es real en las empresas como lo muestra Viles (2007) quien haciendo referencia a un estudio desarrollado en España, demostró que sólo el 50\% de ellas usan herramientas básicas sencillas y pocas involucran herramientas estadísticas avanzadas. Si este comportamiento se presenta en países industrializados, es posible encontrar algo similar o a menor escala en industrias de países en vía de desarrollo.

Lograr el pensamiento probabilístico o aleatorio en las personas es una tarea compleja, que por su importancia tiene inicio desde la educación básica y media con continuidad en la formación universitaria. El pensamiento aleatorio y estadístico fortalece al ingeniero en su razonamiento lógico para la toma de decisiones y la solución de problemas.

La Estadística es una ciencia multidisciplinaria al servicio de todas las profesiones, especialmente de la Ingeniería, la cual por su naturaleza cuantitativa no escapa del manejo de datos y fenómenos permeados por la incertidumbre. Lograr un pensamiento estadístico es un propósito que persigue la universidad pero su evaluación de logro hasta ahora comienza.

La probabilidad no se puede desarticular de la Estadística, ya que es el vínculo para pasar de un análisis descriptivo a uno confirmatorio a través de los procesos inferenciales, los cuales generan resultados con cierta incertidumbre. La Probabilidad y la Estadística apoyan el método de ingeniería para la toma de decisiones empresariales. 


\section{REFERENCIAS BIBLIOGRÁFICAS}

Agnelli, H. (2009). Relevancia de la enseñanza de la Probabilidad. Ciencias Económicas, 2(7), 11-21.

Batanero, C. (2002, febrero). Los retos de la cultura estadística. Conferencia inaugural. Jornadas Interamericanas de Enseñanza de la Estadística. Buenos Aires, Argentina. Recuperado el 15 de agosto de 2012 en http://www.s-a-e.org.ar/revista-vol6.htm

Behar, R., Mesa, E., Díaz, R., Andrade, M., Conde, G., Delgado, J., Klinger, R., Olaya, J. (2002). El Rol de la Estadística en el trabajo del ingeniero. Ingeniería y Competitividad, 4(1), 47 - 54

Carballo, C. y Domínguez, J. (2010). Impulsando el Pensamiento Estadístico con Seis Sigma. Ponencia presentada en XII Seminario de Estadística aplicada IASI. Universidad Nacional de Colombia. Medellín, Colombia.

Cochran, W. (2000). Técnicas de muestreo. (10quinta reimpresión de la 1era ed. en español; A. Sestier Boulier, Trad.). México: Compañía Editorial Continental; S.A. de C.V. (Trabajo original publicado en 1980).

Díaz, L. G. (2002). Estadística multivariada: inferencia y métodos. Bogotá, D. C. Colombia: Panamericana Formas e Impresos S.A.

Guerrero, V. (1991). Análisis estadístico de series de tiempo económicas. Distrito Federal, México: Universidad Autónoma Metropolitana.

Gutiérrez, H., De la Vara, R. (2004). Control estadístico de la calidad y seis sigma. Distrito Federal, México: McGraw-Hill.

Hair, J., Anderson, R., Tatham, R., Black, W. (1999). Análisis multivariante (5ª ed.). Madrid, España: Prentice Hall.

Harrell, C. Ghosh, B. y Bowden, R. (2003). Simulation using promodel (2 $2^{\mathrm{a}}$ ed.). New York, EE.UU.: McGraw-Hill.

Juran, J.y Gryna, F. (1988). Juran’s Quality Control Handbook. (4ªd.). MacGraw-Hill.

Koen, B. (1985). Definition of the engineering method. Washington D.C., EE.UU: American Society for Engineering Education.

León, J. (2007). Enseñando Estadística a futuros ingenieros. Scientia Et Technica, 2(34), 563-567.

López, M. y López, J. (1996). Estadística para actuarios. Madrid, España: MAPFRE. 
Ministerio de Educación Nacional (MEN). (1998). Lineamientos Curriculares de Matemáticas. Serie lineamientos curriculares.Bogotá, Colombia: el autor.

Ministerio de Educación Nacional (MEN). (2006). Estándares Básicos de Competencias en Lenguaje, Matemáticas, Ciencias y Ciudadanas. Bogotá, Colombia: el autor.

Montgomery, D. (1991). Diseño y análisis de experimentos. California, EE.UU.: Grupo Editorial Iberoamérica.

Montgomery, D. y Runge, G. (2004).Probabilidad y Estadística aplicada a la ingeniería. Distrito Federal, México: Limusa S.A.

Navidi, W. (2006). Estadística para ingenieros y científicos. Distrito Federal, México: McGraw-Hill.

Romero, R. y Zúnica, L. (2008). Métodos estadísticos en ingeniería. Distrito Federal, México: Limusa S.A.

Santillan, N. Arcos, J. Vidal, M y Cruz, J. (2009). La Estadística como una herramienta necesaria para los ingenieros geólogos del futuro. Trabajo presentado en el Tercer foro Nacional de Ciencias Básicas. Formación del ingeniero tronco común frente a planes de estudio flexibles 2009. Facultad de ingeniería, Universidad Autónoma de México. México. Recuperado el 03 de julio de 2012 en. http:// dcb.fi-c.unam.mx/Eventos/Foro3/Memorias/temtica_5.html

Viles, E. (2007). Análisis didáctico de la Estadística y la calidad en los estudios de Ingeniería Industrial. Tecnura, 11(21), 54-62.

Willd, C. y Pfannkuch, M. (2007). Statistic Thinking in Empirical Enquiry.International StatisticalReview, 37(3). Doi: 10.1111/j.1751-5823.1999.tb00442

Zapata, C. L. (2011). ¿Cómo contribuir a la alfabetización estadística?. Revista Virtual Universidad Católica del Norte, 33, 234-247. Recuperado el 14 de junio de 2012 en http://revistavirtual.ucn.edu.co/ index.php/RevistaUCN/issue/view/1/showToc 